\title{
Macroscopic on-site evaluation (MOSE) of specimens from solid lesions acquired during EUS-FNB: multicenter study and comparison between needle gauges
}

\section{다(1) $(9)$}

\author{
Authors \\ Benedetto Mangiavillano ${ }^{1,2}$, Leonardo Frazzoni ${ }^{3,4}$, Thomas Togliani ${ }^{5}$, Carlo Fabbri ${ }^{6}$, Ilaria Tarantino ${ }^{7}$, Luca De Luca ${ }^{8}$, \\ Teresa Staiano ${ }^{9}$, Cecilia Binda ${ }^{6}$, Marianna Signoretti ${ }^{6}$, Leonardo H. Eusebi ${ }^{3,4}$, Francesco Auriemma ${ }^{1}$, Laura Lamonaca ${ }^{1}$, \\ Danilo Paduano , Milena Di Leo ${ }^{10}$, Silvia Carrara ${ }^{2,10}$, Lorenzo Fuccio ${ }^{3,4}$, Alessandro Repici2, 10
}

Institutions

1 Gastrointestinal Endoscopy Unit - Humanitas Mater Domini - Castellanza (VA)

2 Humamitas Huniversity

3 Gastroenterology and Endoscopy Unit, IRCCS Azienda Ospedaliero-Universitaria di Bologna, Bologna, Italy

4 Department of Medical and Surgical Sciences, University of Bologna, Bologna, Italy

5 Digestive Endoscopy Unit, ASST Poma Hospital Mantova, Mantova, Italy

6 Endoscopy Unit, Morgagni-Pietrantoni Hospital, ForlìCesena, Italy

7 Endoscopy Service, Mediterranean Institute for Transplantation and Advanced Specialized Therapies (IRCCS - ISMETT), Palermo, Italy

8 Gastroenterology and Digestive Endoscopy Unit, Ospedali Riuniti Marche Nord, Pesaro, Italy

9 Digestive Endoscopy Unit, Candiolo Cancer Institute IRCCS, Candiolo (TO), Italy

10 Humanitas Clinical and Research Center, Digestive Endoscopy Unit, Rozzano (Milan), Italy

submitted 18.12 .2020

accepted after revision $\quad 4.2 .2021$

\section{Bibliography}

Endosc Int Open 2021; 09: E901-E906

DOI 10.1055/a-1395-7129

ISSN 2364-3722

(c) 2021. The Author(s).

This is an open access article published by Thieme under the terms of the Creative Commons Attribution-NonDerivative-NonCommercial License, permitting copying and reproduction so long as the original work is given appropriate credit. Contents may not be used for commercial purposes, or adapted, remixed, transformed or built upon. (https://creativecommons.org/licenses/by-nc-nd/4.0/)

Georg Thieme Verlag KG, Rüdigerstraße 14,

70469 Stuttgart, Germany
Corresponding author

Benedetto Mangiavillano, MD, Gastrointestinal Endoscopy

Unit, Humanitas - Mater Domini, Via Gerenzano n.2, 21053-

Castellanza (VA), Italy

Fax: +00390331476210

bennymangiavillano@gmail.com

\section{ABSTRACT}

Background and study aims The standard method for obtaining samples during endoscopic ultrasonography (EUS) is fine-needle aspiration (FNA), the accuracy of which can be affected by the presence of a cytopathologist in endoscopy room (rapid on-site evaluation [ROSE]). With the introduction of fine-needle biopsy (FNB), macroscopic on-site evaluation (MOSE) of a acquired specimen has been proposed. Only a few studies have evaluated the role of MOSE and in all except one, a 19G needle was used. Our primary aim was to evaluate the diagnostic yield and accuracy of MOSE with different needle sizes and the secondary aim was to identify factors influencing the yield of MOSE. Patients and methods Data from patients who underwent EUS-FNB for solid lesions, with MOSE evaluation of the specimen, were collected in six endoscopic referral centers.

Results A total of 378 patients ( $145 \mathrm{~F}$ and $233 \mathrm{M}$ ) were enrolled. Needles sizes used during the procedures were $20 \mathrm{G}$ (42\%), 22G (45\%), and 25G (13\%). The median number of needle passes was two (IQR 2-3). The overall diagnostic yield of MOSE was of $90 \%$ (confidence interval $[\mathrm{Cl}] 86 \%-$ $92 \%)$. On multivariable logistic regression analysis, variables independently associated with the diagnostic yield of MOSE were a larger needle diameter (20G vs. 25G, OR $11.64,95 \% \mathrm{Cl} 3.5-38.71$; 22G vs. 25G, OR $6.20,95 \% \mathrm{Cl}$ 2.41-15.90) and three of more needle passes (OR 3.39, 95 $\% \mathrm{Cl} 1.38-8.31)$.

Conclusions MOSE showed high diagnostic yield and accuracy. Its yield was further increased if performed with a large size FNB needles and more than two passes. 


\section{Introduction}

Endoscopic ultrasonography (EUS) with tissue acquisition (TA) is a well-established technique for sampling solid lesions pancreatic and non-pancreatic lesions [1-4]. Currently, the standard methods for obtaining samples from gastrointestinal and non-gastrointestinal solid masses is fine-needle aspiration (FNA) performed with EUS. Its sensitivity, specificity, and diagnostic accuracy for malignant cytology range from $78 \%$ to $98 \%$ [5-7]. Several studies have been published in recent years aiming to identify factors related to a non-diagnostic or false-negative EUS-FNA sampling result, and to improve its diagnostic yield using different needle sizes and different tissue acquisition techniques, such as the fanning technique, the slow-pull stylet extraction or the suction technique.

Despite data reporting a high sensitivity and specificity, the diagnostic efficacy of EUS-FNA could be affected by the presence of a cytopathologist in the endoscopy suite during tissue sampling. Rapid on-site evaluation (ROSE) has been advocated to significantly increase EUS-FNA accuracy; however, evidence available in literature is inconsistent. Studies performed with EUS-FNA plus ROSE showed higher sensitivity and similar specificity to those where ROSE was not available; in particular, sensitivity and specificity were $83 \%$ (95\% confidence interval $[\mathrm{Cl}]$, $64 \%-93 \%)$ and $98 \%(95 \% \mathrm{Cl}, 80-100 \%)$ when ROSE was performed, as compared with $65 \%(95 \% \mathrm{Cl}, 57 \%-73 \%)$ and $94 \%$ (95\%Cl, 31\%-100\%) when ROSE was not available [8,9]. Recently, in a systematic review with meta-analysis, was reported as seven on 10 patients who repeated EUS-FNA (rEUS-FNA) for a previous inconclusive diagnosis can receive a correct diagnosis, of which eight with ROSE (80\%) and six without ROSE (60\%). The rEUS-FNA is adequately informative only in case of positive results, whereas in case of negative results, a false negative cannot be reliably excluded [10]. To overcome this issue, following the implementation of fine needle biopsy (FNB) for TA of histological specimens, Iwashita and colleagues introduced for the first time macroscopic on-site evaluation (MOSE) of the acquired tissue performed directly by the endosonographer [11].

The primary aim of our study was to evaluate the diagnostic yield and accuracy of MOSE with different needle sizes, and the secondary aim was to identify factors influencing the yield of MOSE.

\section{Patients and methods}

Data from patients who underwent EUS-FNB for solid lesions, with MOSE evaluation of the specimen, were retrospectively collected in six endoscopic referral centers from January 1 , 2018 to the April 30, 2020. An electronic database was sent to all the participant centers for the data entry. The study received an IRB approval by our institution.

\section{Inclusion and exclusion criteria}

Inclusion criteria were age $>18$ years, both genders; both inpatients and outpatients; presence of a solid lesion and, in the presence of a cystic component, the solid part of the lesion should be more $75 \%$ of the total; and obtained informed consent for EUS with tissue acquisition. Exclusion criteria were patients who underwent previously EUS-FNA with or without ROSE; patients who underwent EUS-FNB plus ROSE; previous biopsy of the lesion with diagnosis of malignancy; presence of an uncorrectable coagulopathy as defined by abnormal prothrombin time (PT) or partial thromboplastin time (PTT) that does not normalize after administration of fresh frozen plasma; an inclusion in an other study.

\section{Endoscopic procedures}

All the EUS procedures were performed by experienced endosonographers with >500 diagnostic EUS and >100 FNA and/or FNB performed, without any specific experience in cytopathology. All of the procedures were performed or under deep sedation with propofol by the anesthetist or under conscious sedation with benzodiazepine plus pethidine administration by the endosonographer. Linear echoendoscopes were used for all the examinations (GF-UCT 140 and GF-UCT180, Olympus, Hamburg, Germany with the corresponding integrated display units EU-ME2 - Premier Plus or with Aloka $\alpha-10$ system; EG3270UK Pentax, Hamburg, Germany). Different sizes of FNB needles were used for the study, specifically 25G, 22G, and $20 \mathrm{G}$ (EchoTip ProCore, Cook Medical, and Acquire, Boston Scientific; Sharkcore, Medtronics). Lesion biopsied were: pancreatic masses, common bile duct (CBD) or gallbladder masses, thoracic and abdominal lymph nodes, esophageal/gastric/duodenal or rectal submucosal lesions, and perirectal lesions. The choice of the needle was decided by the endosonographer based on personal experience and lesion to target. After the needle puncture, the specimens were acquired either with the suction technique (ST) or with the slow-pull technique (SPT). Standard or fanning technique were also used for sampling the lesion [12].

\section{MOSE technique}

After each pass, the stylet was reinserted into the needle to push its content onto a glass slide and allow MOSE of the specimen by the endosonographer. If "worm-like" material $\geq 4 \mathrm{~mm}$ was observed, it was then placed in a $10 \%$ neutral buffered formalin jar for the final histological examination. If bloody material and clots were obtained, they were also collected in formalin. No specimens for cytological examination were collected. After every needle pass the endosonographer evaluated the specimen, judging its adequacy (> Fig.1).

\section{Study outcomes}

The primary outcome of the present study was to evaluate the diagnostic yield and accuracy of MOSE. The diagnostic yield was defined as the proportion of specimens judged adequate by the endosonographer that were confirmed to be adequate also by the pathologist. The diagnostic accuracy of MOSE was defined through sensitivity, specificity, positive and negative predictive value (PPV and NPV). For the purpose of the analysis, we considered malignant lesions as the disease of interest, whereas non-neoplastic and benign lesions were considered as controls. 


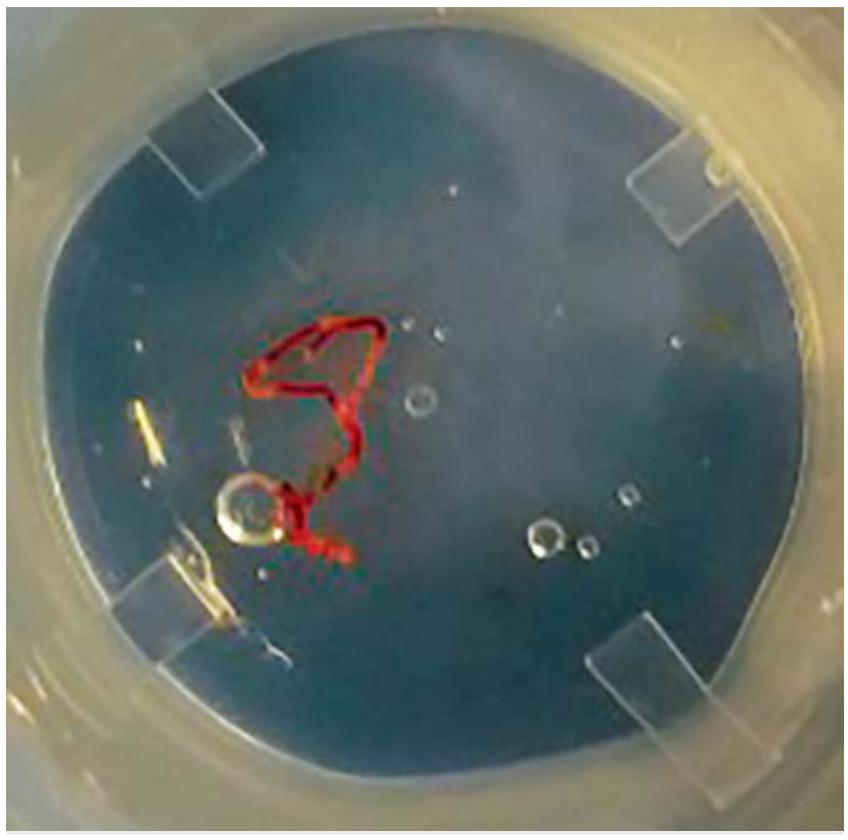

- Fig. 1 Specimen from a pancreatic mass acquired with a $22 \mathrm{G}$ FNB needle (Acquire - Boston Scientific) after one needle pass.

The secondary outcome was to assess and eventually identify variables influencing the diagnostic yield of MOSE.

\section{Statistical analysis and sample size}

Continuous variables were reported as means \pm standard deviations (SDs) when normally distributed, and as medians and interquartile ranges (IQRs) otherwise; categorical variables were described as proportions. Overall accuracy, sensitivity, specificity, positive (PPV) and negative (NPV) predictive value with $95 \%$ confidence interval $(\mathrm{Cl})$ were computed. Samples inadequate for histological evaluation were considered as false negative cases. A multivariable logistic regression analysis was undertaken in order to identify variables independently associated with the diagnostic yield of MOSE. Measures of association were expressed in terms of odds ratio (OR) along with $95 \% \mathrm{Cl}$. $P<0.05$ was considered statistically significant. Estimating from the literature that we would obtain the macroscopic visible core (MVC) as confirmed by the pathologist in around $90 \%$ of cases [13], at least 139 patients would be required for assessing the expected proportion with $5 \%$ absolute precision and $95 \%$ confidence. Statistical analyses were performed with Stata 16 (Stata Corp, College Station, Texas, United States) for Mac.

\section{Results}

Overall, 378 EUS-FNB samples collected at the six centers were judged to be macroscopically adequate by the endosonographers. Mean patient age was $67 \pm 12$ years, whereas 233 patients (62\%) were male. Baseline characteristics of included patients are reported in $>$ Table $\mathbf{1}$. The mean lesion size was $33.8 \pm 17.3 \mathrm{~mm}$. One-hundred seventy lesions (45\%) were pancreatic, whereas 208 lesions (55\%) were extra-pancreatic. A de-
- Table 1 Baseline characteristics of patients who underwent EUSFNB in the study sample.

\begin{tabular}{|l|l|}
\hline & $\begin{array}{l}\text { Study sample } \\
(\mathbf{n = 3 7 8 )}\end{array}$ \\
\hline Patient age, years (mean + SD) & $67+12$ \\
\hline Male sex, $\mathrm{n}(\%)$ & $233(62 \%)$ \\
\hline Lesion size, mm (mean + SD) & $33.8 \pm 17.3$ \\
\hline Lesion site, $\mathrm{n}(\%)$ & \\
\hline Pancreatic & $170(45 \%)$ \\
\hline Extra-pancreatic & $208(55 \%)$ \\
\hline Needle size, $n$ (\%) & \\
\hline $20 \mathrm{G}$ & $157(42 \%)$ \\
\hline $22 \mathrm{G}$ & $171(45 \%)$ \\
\hline $25 \mathrm{G}$ & $50(13 \%)$ \\
\hline EUS-FNB, endoscopic ultrasound-guided fine-needle biopsy. \\
\hline
\end{tabular}

tailed description of the lesion sites is provided in > Table 2. The EUS-FNB needles sizes used for sampling were 20G $(\mathrm{n}=$ $157,42 \%), 22 \mathrm{G}(n=171,45 \%)$, and $25 \mathrm{G}(\mathrm{n}=50,13 \%)$. The EUS-FNB needle types were Procore $(n=218,57 \%$ ), Acquire ( $n$ $=142,38 \%$ ), and others including Echotip ultra and Sharkcore $(n=18,5 \%)$. The median number of needle passes during EUSFNB was two (IQR 2-3).

Three-hundred thirty-nine of 378 samples were judged to be adequate for histological evaluation by the pathologist, with a MOSE overall diagnostic yield of $90 \%$ (95\%Cl 86-92\%). At multivariable logistic regression analysis, variables independently associated with the diagnostic yield of MOSE were a larger needle diameter (20G vs. 25G, OR 11.64, $95 \% \mathrm{Cl} 3.5-38.71 ; 22 \mathrm{G}$ vs. 25G, OR $6.20,95 \% \mathrm{Cl} 2.41-15.90)$ and three or more needle passes (OR 3.39, 95\% Cl 1.38-8.31). Details of this analysis can be found in $>$ Table 3. At histology, 324 (86\%) lesions were malignant. A detailed description of lesion histology is provided in

\section{- Table 2.}

The overall diagnostic accuracy of MOSE for malignancy was $87.3 \%(95 \% \mathrm{Cl}, 83.5-90.5 \%)$, sensitivity was $85.2 \%(95 \% \mathrm{Cl}$, $80.8-88.9 \%)$, specificity was $100 \%$ (95\%Cl, 93.4-100\%), PPV was $100 \%$ (98.7-100\%), and NPV was $52.9 \%(95 \% \mathrm{Cl}, 42.8-$ $62.9 \%$ ), given the $86 \%$ prevalence of malignancy in the study sample. Among pancreatic lesions, the diagnostic accuracy of MOSE for malignancy was $87.6 \%(95 \% \mathrm{Cl}, 81.7 \%-92.2 \%)$, sensitivity was $86.5 \%(95 \% \mathrm{Cl}, 80.2 \%-91.5 \%)$, specificity was $100 \%$ (95\% Cl, 76.8\%-100\%), PPV was $100 \%$ (97.3\%-100\%), and NPV was $40 \%(95 \% \mathrm{Cl}, 23.9 \%-57.9 \%)$, given the $92 \%$ prevalence of malignancy in this group. Among extra-pancreatic lesions, the diagnostic accuracy of MOSE for malignancy was $86.3 \%(95 \% \mathrm{Cl}, 80.9 \%-90.6 \%)$, sensitivity was $83.9 \%(95 \% \mathrm{Cl}$, $77.5 \%-89.1 \%)$, specificity was $100 \%$ (95\%Cl, $91.2 \%-100 \%)$, PPV was $100 \%$ (97.4\%-100\%), and NPV was $59.7 \%(95 \% \mathrm{Cl}, 47$ $\%-71.5 \%$ ), given the $81 \%$ prevalence of malignancy in this group. Among 69 subepithelial lesions (SELs) distributed along- 
- Table 2 Lesion characteristics and diagnostic yield and final histological diagnosis displayed according to pancreatic and extra-pancreatic site.

\begin{tabular}{|c|c|c|c|}
\hline Lesion site & n (\%) & Diagnostic yield, n (\%) & Final diagnosis \\
\hline Pancreatic & $170(45 \%)$ & $156(92 \%)$ & \multirow{5}{*}{$\begin{array}{l}134 \text { DACs } \\
18 \text { NETs } \\
10 \text { chronic pancreatitis } \\
3 \text { metastases } \\
2 \text { pseudopapillary tumor } \\
\text { Other }(n=3)^{1}\end{array}$} \\
\hline Uncinate & $16(4 \%)$ & & \\
\hline Head & $107(29 \%)$ & & \\
\hline Body & $38(10 \%)$ & & \\
\hline Tail & $9(2 \%)$ & & \\
\hline Extra-pancreatic & $208(55 \%)$ & $183(88 \%)$ & \multirow{10}{*}{$\begin{array}{l}89 \text { adenocarcinomas } \\
29 \text { GISTs } \\
26 \text { lymphomas } \\
12 \text { benign lymphocytes } \\
7 \text { sarcoidosis } \\
5 \text { leiomyomas } \\
4 \text { each for germ cell tumor, SCC, and benign gastric cells } \\
3 \text { NETs } \\
3 \text { reactive lymph nodes } \\
3 \text { Leiomyosarcomas } \\
\text { Other }(n=19)^{2}\end{array}$} \\
\hline Esophagus & $5(1 \%)$ & & \\
\hline Stomach & $53(15 \%)$ & & \\
\hline Duodenum & $11(3 \%)$ & & \\
\hline Abdominal lymph nodes & $61(16 \%)$ & & \\
\hline Mediastinum & $43(11 \%)$ & & \\
\hline CBD or gallbladder & $12(3 \%)$ & & \\
\hline Rectal or peri-rectal & $16(4 \%)$ & & \\
\hline Liver & $5(1 \%)$ & & \\
\hline Other ${ }^{3}$ & $2(1 \%)$ & & \\
\hline \multicolumn{4}{|c|}{$\begin{array}{l}\text { DAC, ductal adenocarcinoma; FN, false negative; IPMN, intraductal papillary mucinous neoplasm; NET, neuroendocrine tumor; SCC, squamous cell cancer; SMT, } \\
\text { submucosal tumor. } \\
{ }^{1} \mathrm{n}=1 \text { each for IPMN, mucinous cystoadenocarcinoma, and accessory spleen. } \\
{ }^{2} \text { Small cell lung cancer, tuberculosis, Schwannoma, renal cell cancer ( } \mathrm{n}=2 \text { for each); benign biliary cells, HCC, endometrial cancer, uterine cervical cancer, meso- } \\
\text { thelioma, ovarian cancer, melanoma, pancreatic rest, paraganglioma, glomic tumor, haemangioma. } \\
{ }^{3} \mathrm{n}=1 \text { each for left adrenal gland and spleen. }\end{array}$} \\
\hline
\end{tabular}

side the gastrointestinal tract, the diagnostic accuracy of MOSE for malignancy was $81.2 \%(95 \% \mathrm{Cl}, 69.9 \%-89.6 \%)$, sensitivity was $76.4 \%$ (95\%Cl, $63 \%-86.8 \%)$, specificity was $100 \%(95 \% \mathrm{Cl}$, $76.8 \%-100 \%)$, PPV was $100 \%(95 \% \mathrm{Cl}, 91.6 \%-100 \%)$, and NPV was $51.9 \%(95 \% \mathrm{Cl}, 31.9 \%-71.3 \%)$, given the $80 \%$ prevalence of malignancy in this group.

\section{Discussion}

The MOSE of the acquired EUS specimen by FNB needle is a relatively new easy technique aiming to improve the sampling diagnostic accuracy and to diminish costs related to the presence of a cytopathologist and its instrumentation in the endoscopy suite. The first report of the MOSE technique was published in 2015 from a Japanese group in a cohort of patients who underwent EUS-FNA with a 19G needle. The Authors showed a diagnostic accuracy of $78.9 \%$, and also observed that a macroscopic visible core (MVC) $\geq 4 \mathrm{~mm}$ on MOSE can be an indicator of specimen adequacy and can improve the diagnostic yield [11]. However, ROSE performed by endosonographer is also possible [14], and a recent meta-analysis showed that FNB outperformed FNA when sampling pancreatic and nonpancreatic lesions. Moreover, the authors found that forward-facing bevel FNB needles seemed to outperform the reverse bevel FNB needles [15]. To evaluate the role of ROSE during FNB sampling, a multicenter, randomized, non-inferiority trial was conducted in 16 international centers, including a large prospective cohort of
- Table 3 Multivariable logistic regression analysis to assess variables associated with the diagnostic yield of MOSE.

\begin{tabular}{|l|l|l|}
\hline Variables & Odds ratio (95\%Cl) & P value \\
\hline High-vs. low-volume center & $0.32(0.07-1.36)$ & 0.123 \\
\hline Needle type & & \\
\hline Procore & Ref. & - \\
\hline Acquire & $2.40(0.88-6.53)$ & 0.088 \\
\hline Others (Echotip, Sharkcore) & $0.98(0.11-8.93)$ & 0.983 \\
\hline Needle diameter & & \\
\hline $20 G$ & $11.64(3.5-38.71)$ & 0.001 \\
\hline $22 G$ & $6.20(2.41-15.90)$ & 0.001 \\
\hline $25 G$ & Ref. & - \\
\hline $\begin{array}{l}\text { Pancreatic vs. extra-pancreatic } \\
\text { site }\end{array}$ & $1.86(0.87-3.95)$ & 0.108 \\
\hline$\geq 3$ needle passes & $3.39(1.38-8.31)$ & 0.008 \\
\hline Lesion diameter & $1.37(0.68-2.76)$ & 0.380 \\
\hline MOSE, macroscopic on-site evaluation. & \\
\hline
\end{tabular}

patients. The authors randomized 800 patients with solid pancreatic masses to sampling with EUS-FNB plus ROSE or to EUSFNB alone, demonstrating the non-inferiority of FNB alone [16]. 
With the advent of FNB, MOSE of the specimen by the endosonographer has been proposed as an alternative to ROSE [17], but only few studies are reported in literature about this issue.

To our knowledge, our multicenter series is the largest one in the current literature. Our results showed that MOSE had an $89 \%$ overall diagnostic yield, with a median number of needle passes during EUS-FNB of two. Analyzing our results, we found that variables independently associated with the diagnostic yield of MOSE were the larger needle diameter and three or more needle passes. It could be hypothesized that the thicker the specimen is, the more likely the gross appearance would be judged as adequate by the pathologist. This result appears to be clinically relevant, as it supports the use of a large-caliber needles when ROSE is not feasible. Moreover, it is plausible that more needle passes lead to more tissue acquired, increasing the likelihood of a correct diagnosis. Diagnostic accuracy, sensitivity, specificity, PPV and NPV for pancreatic and extra-pancreatic masses were comparable, enlightening that the diagnostic accuracy of the MOSE does not seem to be affected by the target lesions but only by needle diameter and number of passes.

A recent RCT with a total of 244 patients who underwent EUS plus fine needle tissue acquisition (FNTA) with a 19G needle showed that the diagnostic yield of the MOSE technique (92.6\%) was similar to the conventional technique $(89.3 \% ; P=$ 0.37 ), requiring significantly fewer needle passes (median: conventional 3, MOSE 2; $P<0.001$ ) [18]. The diagnostic yield reported in this RCT was slightly higher than ours. This could be explained by the use of only 19G FNTA needles, whereas is our series the overall accuracy was evaluated using 20G, 22G and $25 \mathrm{G}$ needles. Moreover, one of the inclusion criteria of the RCT was a lesion diameter $>2 \mathrm{~cm}$, while in our study there were no restrictions based on the lesion size. A recent retrospective study including a cohort of 54 patients who underwent EUS-FNB with a 22G Franseen-tip needle, demonstrated that MOSE could limit the number of needle passes by accurately evaluating the histologic core fragments, showing a diagnostic adequacy and accuracy of more than $90 \%$ [19].

Our study has some limitations. First, because of the retrospective design, we could not retrieve data on the tissue acquisition technique used to acquire the specimen. Different techniques have been proposed for targeting lesions during TA. The standard technique (ST) consists in placing the tip of the needle within the lesion, moving the needle to-and-fro on the same axis, regardless of the TA technique applied (standard suction, slow-pull, wet or non-suction technique). Two other techniques, such as the fanning technique (FT) and the torque technique (TT) have also been proposed to establish an accurate diagnosis with fewer needle passes $[20,21]$ showing different results. No study comparing the FT vs TT has been published yet. Nevertheless, most endosonographers combine these three techniques based on their personal experience and on the characteristic of the lesion to target. Second, the multivariable analysis provided quite large $\mathrm{Cls}$, decreasing the confidence in the estimates. However, the significant variables (i.e. increasing number of passes and larger needle size), although predictable, provide insightful information for clinical practice.

\section{Conclusions}

In conclusion, MOSE during EUS-FNB sampling showed high diagnostic yield and accuracy using different needle sizes, adding new evidence to current literature, as most published studies used MOSE in combination with $19 \mathrm{G}$ needles. The diagnostic yield was higher when FNB was performed with larger-caliber needles (i.e. 22G or 20G) and with more than two passes. Therefore, this technique may represent a valid alternative when ROSE is not feasible. Studies comparing EUS-FNA plus ROSE vs EUS-FNB plus MOSE could establish if the two techniques are similar in terms of diagnostic efficacy. Moreover, studies comparing EUS-FNB plus MOSE vs EUS-FNB with standard technique with smaller diameter needles (22G or 25G) could also add valuable clinical data. Finally, interactive cytopathology training of endosonographers could further improve the accuracy of the MOSE technique.

\section{Competing interests}

The authors declare that they have no conflict of interest.

\section{References}

[1] Chang KJ, Nguyen P, Erickson RA et al. The clinical utility of endoscopic ultrasound-guided fine-needle aspiration in the diagnosis and staging of pancreatic carcinoma. Gastrointest Endosc 1997; 45: 387393

[2] Varadarajulu S, Eloubeidi MA. The role of endoscopic ultrasonography in the evaluation of pancreaticobiliary cancer. Surg Clin N Am 2019; 90: 251-263

[3] Dewitt J, Devereaux BM, Lehman GA et al. Comparison of endoscopic ultrasound and computed tomography for the preoperative evaluation of pancreatic cancer: a systematic review. Clin Gastroenterol Hepatol 2006; 4: 717-725

[4] Chhieng DC, Jhala D, Jhala N et al. Endoscopic ultrasound-guided fineneedle aspiration biopsy: a study of 103 cases. Cancer. Cancer Cytopathol 2003; 96: 232-239

[5] Ngamruengphong S, Li F, Zhou Y et al. EUS and survival in patients with pancreatic cancer: a population-based study. Gastrointest Endosc 2010; 72: 78-83

[6] Othman MO, Wallace MB. The role of endoscopic ultrasonography in the diagnosis and management of pancreatic cancer. Gastroenterol Clin North Am 2012; 41: 179-188

[7] Attila T, Aidin Ö. Lesion size determines diagnostic yield of EUS-FNA with onsite cytopathologic evaluation for upper gastrointestinal subepithelial lesions. Turk J Gastroenterol 2018; 29: 436-441

[8] Kappelle WFW, Van Leerdam ME, Schwartz MP et al. Rapid on-site evaluation during endoscopic ultrasound-guided fine-needle aspiration of lymph nodes does not increase diagnostic yield: A randomized, multicenter trial. Am J Gastroenterol 2018; 113: 677-685

[9] Fabbri C, Fuccio L, Fornelli A et al. The presence of rapid on-site evaluation did not increase the adequacy and diagnostic accuracy of endoscopic ultrasound-guided tissue acquisition of solid pancreatic lesions with core needle. Surg Endosc 2017; 31: 225-230

[10] Lisotti A, Frazzoni L, Fuccio L et al. Repeated EUS-FNA of pancreatic masses after nondiagnostic or inconclusive results: systematic review and meta-analysis. Gastrointest Endosc 2020; 91: 1234-1241 
[11] Iwashita T, Yasuda I, Mukai T et al. Macroscopic on-site quality evaluation of biopsy specimens to improve the diagnostic accuracy during EUS-guided FNA using a 19-gauge needle for solid lesions: a single-center prospective pilot study (MOSE study). Gastrointest Endosc 2015; 81: 177-185

[12] Mangiavillano B, Sosa-Valenca L, Deprez P et al. Tissue acquisition and pancreatic masses: Which needle and which acquisition technique should be used? Endosc Int Open 2020; 8: E1315-E1320

[13] Oh D, Seo DW, Hong SM et al. The impact of macroscopic on-site evaluation using filter paper in EUS-guided fine-needle biopsy. Endosc Ultrasound 2019; 8: 342-347

[14] Hikichi T, Irisawa A, Bhutani MS et al. Endoscopic ultrasound-guided fine-needle aspiration of solid pancreatic masses with rapid on-site cytological evaluation by endosonographers without attendance of cytopathologists. J Gastroenterol 2009; 44: 322-328

[15] van Riet PA, Erler NS, Bruno MJ et al. Comparison of fine-needle aspiration and fine-needle biopsy devices for endoscopic ultrasoundguided sampling of solid lesions: a systemic review and meta-analysis. Endoscopy 2020: doi:10.1055/a-1206-5552 (Online ahead of print.)

[16] Crinò SF, Manfrin E, Scarpa A et al. EUS-FNB with or without on-site evaluation for the diagnosis of solid pancreatic lesions (FROSENOR):
Protocol for a multicenter randomized non-inferiority trial. Dig Liver Dis 2019; 51: 901-906

[17] Han P, Che D, Pallav K et al. Models of the cutting edge geometry of medical needles with applications to needle design. Int J Mech Sci 2012; 65: 157-167

[18] Chong CCN, Lakhtakia S, Nguyen N et al. Endoscopic ultrasoundguided tissue acquisition with or without macroscopic on-site evaluation: randomized controlled trial. Endoscopy 2020; 52: 856-863

[19] Leung KiEL, Lemaistre AI, Fumex F et al. Macroscopic onsite evaluation using endoscopic ultrasound fine needle biopsy as an alternative to rapid onsite evaluation. Endosc Int Open 2019; 7: E189-E194

[20] Bang JY, Magee SH, Ramesh J et al. Randomized trial comparing fanning with standard technique for endoscopic ultrasound-guided fineneedle aspiration of solid pancreatic mass lesions. Endoscopy 2013; 45: $445-450$

[21] Park SW, Lee SS, Song T] et al. The diagnostic performance of novel torque technique for endoscopic ultrasound-guided tissue acquisition in solid pancreatic lesions: A prospective randomized controlled trial. Gastroenterol Hepatol 2020; 35: 508-515 